\title{
An Automated Method to Compute Orbital Re-entry Trajectories with
}

\author{
Heating Constraints
}

\author{
Curtis Zimmerman, Greg Dukeman, and John Hanson \\ NASA Marshall Space Flight Center \\ Huntsville, AL 35812
}

Determining how to properly manipulate the controls of a re-entering re-usable launch vehicle (RLV) so that it is able to safely return to Earth and land involves the solution of a two-point boundary value problem (TPBVP). This problem, which can be quite difficult, is traditionally solved on the ground prior to flight. If necessary, a nearly unlimited amount of time is available to find the "best" solution using a variety of trajectory design and optimization tools. The role of entry guidance during flight is to follow the pre-determined reference solution while correcting for any errors encountered along the way. This guidance method is both highly reliable and very efficient in terms of onboard computer resources. There is a growing interest in a style of entry guidance that places the responsibility of solving the TPBVP in the actual entry guidance flight software. Here there is very limited computer time. The powerful, but finicky, mathematical tools used by trajectory designers on the ground cannot in general be made to do the job. Nonconvergence or slow convergence can result in disaster. The challenges of designing such an algorithm are numerous and difficult. Yet the payoff (in the form of decreased operational costs and increased safety) can be substantial. This paper presents an algorithm that incorporates features of both types of guidance strategies. It takes an initial RLV orbital re-entry state and finds a trajectory that will safely transport the vehicle to a Terminal Area Energy Management (TAEM) region. During actual flight, the computed trajectory is used as the reference to be flown by a more traditional guidance method.

\section{Introduction}

In the design of entry trajectories, two control variables are normally available: alpha (angle of attack) and phi (bank angle) ${ }^{1}$. The entry guidance algorithm developed in this paper (referred to as EGuide) primarily attempts to find and adjust bank angle profiles to meet final state constraints while maintaining a constant or pre-determined angle of attack profile. The key feature that makes EGuide unique from traditional guidance methods is its ability to solve trajectory problems onboard. Although research into algorithms with numerical solution capability is not new ${ }^{2-4}$, their use as actual flight software remains untapped. This is due mainly 
to issues of computational complexity that accompany all numerical procedures of this nature. EGuide addresses some of these issues with an architecture that combines the best of traditional guidance methods with the advantage of onboard solution capability. EGuide uses a classic shooting method as its solver. When solving a specific entry problem, EGuide adjusts parameters to meet specified goals using a Newton method that has been configured to generate its Jacobian matrix by flying predictive simulations. For orbital re-entry, most of the heavy computational load of the Newton process can occur prior to the de-orbit burn. This is what is meant by an orbital entry planner. The solution derived on-board can be used to supply trajectory information to a more traditional profile-following guidance law such as Dukeman's $\mathrm{LQR}^{5}$. It is also a natural setup to run as a predictor/corrector guidance. EGuide contains a planning stage and functions as guidance with a combination of a predictor/corrector and a profile- follower using LQR. Development and testing of EGuide has been carried out using the Marshall Aerospace Vehicle representation in "C" (MAVERIC) simulation. MAVERIC is a full 6-DOF simulation developed to test GNC algorithms for the X33.

\section{Shooting Method}

EGuide solves the TPBVP using a version of mnewt ${ }^{6}$ that has been adapted to allow for the limited behavior of an RLV. The mnewt Jacobian matrix is generated by measuring the effect of control changes on the final state of simulated flights. EGuide uses a self-contained 3-DOF trajectory simulation (independent from MAVERIC) that models motion over a rotating oblate Earth with a US62 standard atmosphere. The equations of motion are integrated using a $4^{\text {th }}$ order Runge-Kutta algorithm with a fixed step size of 1 second. The vehicle model includes attitude rate and acceleration limiting to more accurately depict maneuvers during flight. 


\section{Sub-Orbital Entry Guidance}

Part of EGuide is dedicated to solving the TPBVP of a sub-orbital entry trajectory. Specifically, it tries to figure out how to deliver a vehicle from a variety of widely dispersed suborbital entry conditions to a TAEM interface box within an acceptable tolerance of altitude, range, and heading error.

To solve the sub-orbital problem, a linear equation is used to define the bank angle. At each time point during an EGuide trajectory simulation the commanded bank angle is computed using the following formulation:

$$
\varphi_{c m d}=\operatorname{abs}\left(\varphi_{\text {slope }}+\varphi_{i}\left[t_{c u r r e n t}-t_{\text {init }}\right]\right)
$$

where $t_{\text {current }}$ is the current simulated time point, and $t_{\text {init }}$ is the time at the beginning of the simulated trajectory. The sign of $\varphi_{c m d}$ is assigned separately to maintain the heading angle within a specified corridor. Alpha angle is pre-defined as a function of Mach number. Through shooting, EGuide identifies the individual values that parameters $\varphi_{\mathrm{i}}$ and $\varphi_{\text {slope }}$ must take so that the vehicle will fly to TAEM.

For a nominal X33 sub-orbital flight, the EGuide planning phase takes place shortly after main engine cutoff (MECO). Once parameters $\varphi_{i}$ and $\varphi_{\text {slope }}$ from equation (1) have been found, the trajectory is recorded and LQR is activated to guide the vehicle to TAEM. Solving a suborbital entry trajectory problem is essential in the process that EGuide uses to solve for an orbital re-entry trajectory. It is also a required function to participate in the Advanced Guidance and Control Project ${ }^{7}$. This project uses the MAVERIC simulation loaded with an X33 vehicle model and the Global Reference Atmospheric Model (GRAM) as its testing platform and provides a 
scored evaluation to the participating algorithms. 3-DOF and 6-DOF nominal and off-nominal X33 missions are included in the testing criteria.

\title{
Sub-Orbital Results
}

Two X33 missions are used for guidance algorithm testing in the Advanced Guidance and Control Project. The first mission represents a nominal trajectory, while the second uses a higher energy trajectory. Both missions launch from Edwards Air Force Base, CA and land at Michael Army Airfield, Dugway Proving Ground, UT. Results of 100 dispersed flights are shown in Table 1. Dispersion quantities include variables relating to engine performance, aerodynamic uncertainty, navigation accuracy, and vehicle mass properties, as well as atmospheric density and wind dispersions. In addition to the nominal missions, flights with exceptional circumstances are also included in the testing criteria. Large thrust dispersions simulate mis-modeled engine performance. Power pack out (PPO) cases simulate losing 50\% of nominal engine thrust at various times during ascent. Results for these flights are shown in Table 2. TAEM objectives for all missions are:

\author{
Range to $\mathrm{HAC}^{*}$ at $\mathrm{TAEM}=30 \mathrm{~nm} \pm 6 \mathrm{~nm}$ \\ Relative Heading to $\mathrm{HAC}^{*}$ at $\mathrm{TAEM}=0 \mathrm{deg} \pm 10 \mathrm{deg}$ \\ Altitude at $\mathrm{TAEM}=96000 \mathrm{ft} \pm 6000 \mathrm{ft}$ \\ TAEM velocity $=3000 \mathrm{ft} / \mathrm{s}$
}

*Heading Alignment Circle - a latitude, longitude target. 


\begin{tabular}{|c|c|c|c|c|}
\hline Mission X33-1 & Min & Max & Average & Stdev. \\
\hline Range (nm) & 27.39 & 32.38 & 30.24 & 1.14 \\
\hline Heading (deg) & 0.94 & 10.19 & 3.72 & 1.70 \\
\hline Altitude (ft) & 93846 & 97695 & 95837 & 943 \\
\hline Iterations & 2 & 4 & 2.8 & 0.42 \\
\hline
\end{tabular}

\begin{tabular}{|c|c|c|c|c|}
\hline Mission X33-2 & Min & Max & Average & Stdev. \\
\hline Range (nm) & 28.55 & 31.92 & 30.86 & 0.56 \\
\hline Heading (deg) & -1.54 & 9.15 & 2.27 & 2.30 \\
\hline Altitude (ft) & 93803 & 101853 & 98099 & 1878 \\
\hline Iterations & 3 & 9 & 4.47 & 1.87 \\
\hline
\end{tabular}

Table 1. Dispersion Results for 100 6-DOF simulations of two X33 missions.

\begin{tabular}{|c|c|c|c|c|}
\hline Mission & Range (nm) & Alt (ft) & Heading (deg) & Iterations \\
\hline 40 sec. PPO & -26.92 & 100179 & -0.50 & 4 \\
\hline 50 sec. PPO & 29.75 & 95764 & 6.25 & 3 \\
\hline 60 sec. PPO & 29.95 & 96657 & 0.61 & 3 \\
\hline 112 sec. PPO & 28.27 & 97209 & 2.93 & 4 \\
\hline +4 Sigma Thrust & 25.82 & 97552 & 6.69 & 4 \\
\hline +6 Sigma Thrust & 26.22 & 97620 & 8.74 & \\
\hline
\end{tabular}

Table 2. Results for 3-DOF Mission X33-1 off-nominal cases. 


\section{Constant Heat-Rate Tracking}

Although equation (1) can be used to generate valid trajectories from orbital re-entry states, it does not contain enough flexibility to consistently return practical solutions. Heat and dynamic pressure constraints, which have previously been ignored, must somehow be addressed to assure safe flight conditions. Heating in particular can be effectively controlled using a bank angle formulation designed to maintain a constant heat rate during flight in the relevant portion of atmospheric entry.

\section{Equations of Motion}

From the 3-DOF equations of motion for a vehicle flying over a spherical Earth we have ${ }^{8}$ :

$$
\begin{gathered}
\dot{\mathrm{r}}=\mathrm{V} \sin \gamma \\
\dot{\mathrm{V}}=-\mathrm{D}-\frac{\mu}{\mathrm{r}^{2}} \sin \gamma \\
\dot{\gamma}=\frac{1}{\mathrm{~V}}\left\{\mathrm{~L} \cos \sigma+\left(\mathrm{V}^{2}-\frac{\mu}{\mathrm{r}}\right) \frac{\cos \gamma}{\mathrm{r}}+2 \omega \mathrm{V} \cos \varphi \cos \phi\right\}
\end{gathered}
$$

where $r$ is the radial distance from the center of the Earth to the vehicle, $V$ is the Earth-relative velocity magnitude of the vehicle, $\varphi$ is the azimuth of $V$ measured clockwise from North, $\gamma$ is the Earth-relative flight path angle, $\phi$ is the latitude, $\sigma$ is the vehicle bank angle, $\omega$ is the rotation rate of the Earth, and $\mu$ is the gravitational constant. L and D represent aerodynamic lift and drag forces and are given by : $L=1 / 2 m \rho V^{2} S_{\text {ref }} C_{L}, D=1 / 2 m \rho V^{2} S_{\text {ref }} C_{D}$ where $m$ is vehicle mass and $S_{\text {ref }}$ is the vehicle reference area. $C_{L}$ and $C_{D}$ are the lift and drag coefficients of the vehicle and are 
functions of the angle of attack and Mach number. The atmospheric density $\rho$ is approximated by the exponential expression: $\rho=\rho_{0} \mathrm{e}^{-\beta \mathrm{h}}$ where $\rho_{0}$ and $\beta$ are constants, and $\mathrm{h}$ is the altitude.

\section{Heat-Rate Control}

For a vehicle entering a planetary atmosphere, the time rate of average heat input per unit area can be estimated with the expression ${ }^{9}$

$$
\dot{Q}=C \sqrt{\rho} V^{n}
$$

where $\mathrm{n}=3.15$, and $\mathrm{C}$ is a constant. Heat-rate tracking guidance begins with the definition of an error term

$$
\mathrm{e}=\dot{\mathrm{Q}}-\dot{\mathrm{Q}}_{\text {ref }}
$$

where $\dot{Q}_{\text {ref }}$ is a reference heat rate. The intent is for the error term to exhibit the behavior of a stable second order feedback system. To accomplish this, it is substituted into the following classical second order system

$$
\ddot{\mathrm{e}}+2 \zeta \omega_{\mathrm{n}} \dot{\mathrm{e}}+\omega_{\mathrm{n}}^{2} \mathrm{e}=0
$$

where $\zeta$ and $\omega_{\mathrm{n}}$ are constants. The three preceding equations along with the equations of motion yield the following bank angle formulation:

$$
\begin{gathered}
\varphi_{\mathrm{cmd}}=\cos ^{-1}\left\{\frac{\left(\dot{\gamma}_{\mathrm{cmd}}-\frac{\mathrm{V}}{\mathrm{r}}+\frac{\overline{\mathrm{g}}}{\mathrm{v}}\right) \frac{\mathrm{V}}{\mathrm{D}}}{\left(\frac{\mathrm{L}}{\mathrm{D}}\right)_{\mathrm{est}}}\right\} \\
\dot{\gamma}_{\mathrm{cmd}}=\frac{-\mathrm{a}-2 \zeta \omega_{\mathrm{n}} \ddot{\mathrm{Q}}-\omega_{\mathrm{n}}^{2}\left(\dot{\mathrm{Q}}-\dot{\mathrm{Q}}_{\mathrm{ref}}\right)}{\mathrm{b}}
\end{gathered}
$$

where $\mathrm{a}$ and $\mathrm{b}$ are expressions that are determined in the second time derivative of equation (3) and $\overline{\mathrm{g}}$ is the acceleration due to gravity. Thus, given a reference heat-rate $\dot{\mathrm{Q}}_{\mathrm{ref}}$ and using 
equation (6) to generate bank angle control commands, the vehicle is expected to track a constant heat rate.

\section{Orbital Re-entry Planning}

In an orbital re-entry, high heating starts near the beginning of the flight back into the atmosphere when speeds are still close to orbital velocity. Re-entry guidance can begin as soon as there is enough dynamic pressure to maintain adequate control of the vehicle. The EGuide planning simulation is configured to use heat rate tracking guidance at the onset of orbital reentry. Once the vehicle has been safely transported through high heating, heat rate tracking guidance is deactivated and the sub-orbital guidance formulation is used for the remainder of the flight. The trajectory is fully characterized by four parameters (Fig. 1). The first three parameters $\dot{\mathrm{Q}}_{\text {ref }}, \varphi_{\mathrm{i}}$, and $\varphi_{\text {slope }}$ are from the heat rate tracking and sub-orbital guidance formulations. The fourth parameter is the time chosen to terminate heat rate tracking and switch to sub-orbital guidance and is referred to as the guidance switch time (GSWT).

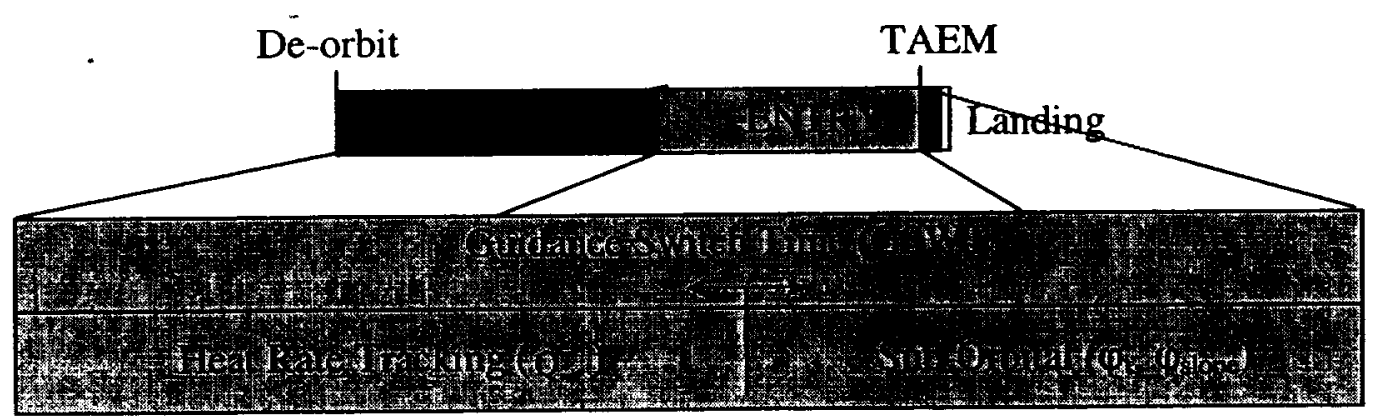

Figure 1. The four parameters of an EGuide orbital re-entry trajectory. 


\section{Finding the Guidance Switch Time (GSWT)}

Calculation of the GSWT parameter is based on observed bank angle behavior during heat rate tracking. As illustrated in Figure 2, in the very first part of an orbital re-entry, heat rate tracking guidance may modulate the bank angle quickly as the control "latches on" to the specified reference heat rate. This initial transient behavior gives way to a slower varying bank angle which is decreasing in magnitude as the control tracks the reference. If heat rate tracking is allowed to remain active indefinitely, the magnitude of the bank angle eventually begins to increase and finally becomes excessive as the control struggles to track a reference heat rate it can no longer sustain. The main feature of interest in the bank angle vs. time plot of Figure 2 is the local minimum that occurs at approximately 800 seconds (Point $\mathrm{A}$ ). Point $\mathrm{A}$ is designated as the GSWT. In actuality, good solutions to the orbital re-entry problem may exist by switching guidance formulations anywhere along the bank angle profile between points $\mathrm{A}$ and $\mathrm{B}$.
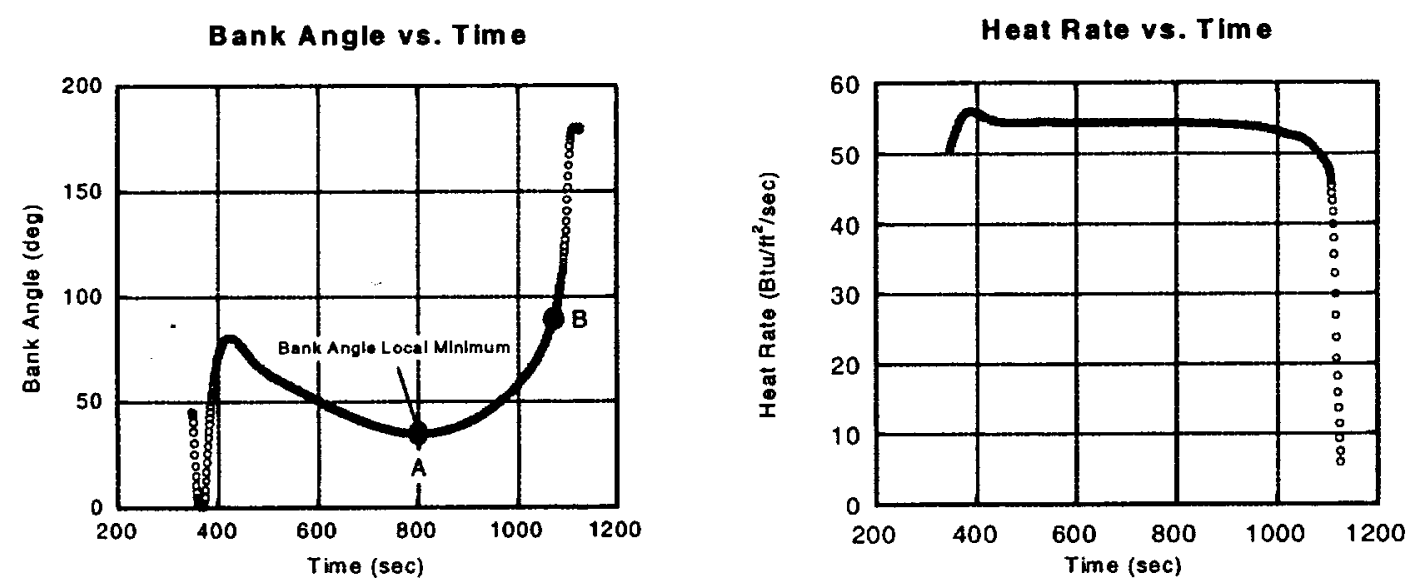

Figure 2. Heat rate tracking bank angle profile with the corresponding heat rate profile.

However, Point A offers the advantage of being an event that is easily detectable. EGuide is programmed to find Point A by simulating an orbital re-entry (using heat rate tracking guidance 
exclusively) and looking for the last occurrence of a bank angle minimum. The fact that the bank angle magnitude must increase after Point $\mathrm{A}$ to maintain the reference heat rate indicates that the high heating portion of the flight has passed.

\section{Setting the Reference Heat-rate Parameter}

The reference heat-rate parameter $\dot{\mathrm{Q}}_{\text {ref }}$ can be thought of as the independent variable of an EGuide orbital re-entry problem. The three other variable parameters GSWT, $\varphi_{i}$, and $\varphi_{\text {slope }}$ are all essentially dependant on the selection of $\dot{\mathrm{Q}}_{\text {ref }}$. Solving the orbital re-entry problem boils down to identifying an appropriate reference heat-rate value that will (in combination with the three remaining variable parameters) result in acceptable behavior for the duration of the trajectory. The search for $\dot{Q}_{\text {ref }}$ is bounded. The lower bound is found by observing what reference heat-rate, when tracked for the duration of a simulated flight, results in the least range error from the TAEM target. The upper bound can be set from actual vehicle heat-rate constraints, although it may be possible to simulate a successful orbital re-entry trajectory at a higher heat-rate than the vehicle can withstand. With upper and lower bounds set for the reference heat-rate parameter, EGuide can begin the search for the solution to the orbital re-entry problem. Starting with $\dot{\mathrm{Q}}_{\text {ref }}$ at its lower bound, a three step iterative process is used:

1). Compute the guidance switch time based on the reference heat-rate.

2). Using the sub-orbital guidance formulation, solve the TPBVP that starts at the guidance switch time (Point A) and ends at TAEM. 
3). Evaluate the solution: If any part of the line segment defined by the sub-orbital guidance parameters $\varphi_{\mathrm{i}}$, and $\varphi_{\text {slope }}$ is above the constant heat-rate curve, the chosen reference heat-rate will be exceeded during the sub-orbital guidance portion of the flight (Fig 3). This is an indication that the reference heat rate is set too low. EGuide performs a limited version of this test by comparing the value of $\varphi_{i}$ with the local minimum of the bank angle at Point $A$. If $\varphi_{i}$ is greater than the bank angle value at Point $\mathrm{A}$, the reference heat rate is increased by one unit and the process is repeated starting at step 1). Convergence of the process occurs when $\varphi_{i}$ is less than or equal to the local minimum of the bank angle at Point $\mathrm{A}$ or, in other words, when the reference heat rate is not exceeded during sub-orbital guidance.

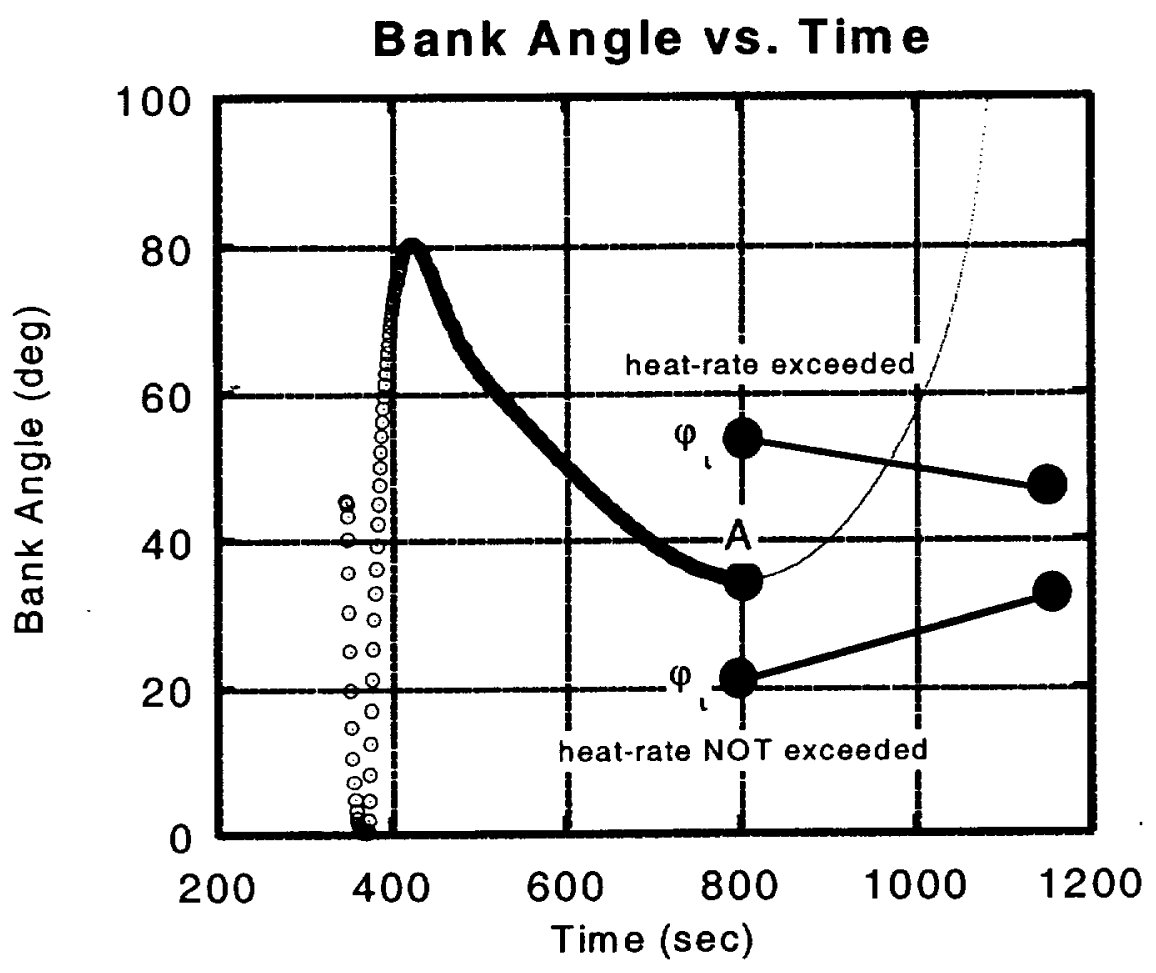

Figure 3. Evaluation of the sub-orbital guidance solution 


\title{
Orbital Re-entry Flight
}

Once the orbital re-entry planner has converged to a good solution, the vehicle can be flown from its initial orbital re-entry state to TAEM. EGuide uses heat-rate tracking guidance during the initial portion of re-entry rather than handing off the guidance responsibility to a profile following method. The main advantage to this strategy is the direct influence over heat-rate through feedback control. At the guidance switch time EGuide acts as a predictor-corrector by performing a single update to the sub-orbital guidance parameters before recording the final trajectory and handing off the results to be flown by LQR guidance.

\section{Orbital Re-entry Results}

Results from six orbital re-entry missions flown with the X33 vehicle are shown. Missions 1-3 are low, high right, and high left cross-range cases which re-enter from an orbital inclination of 51.6 degrees. Missions 4-6 are low, high right, and high left cross-range cases which re-enter from an orbital inclination of 28.5 degrees. All missions land at the Kennedy Space Center after reaching a TAEM target with the same objectives as the sub-orbital flights:

\author{
Range to $\mathrm{HAC}$ at $\mathrm{TAEM}=30 \mathrm{~nm} \pm 6 \mathrm{~nm}$ \\ Relative Heading to $\mathrm{HAC}$ at $\mathrm{TAEM}=0 \mathrm{deg} \pm 10 \mathrm{deg}$ \\ Altitude at TAEM $=96000 \mathrm{ft} \pm 6000 \mathrm{ft}$ \\ TAEM velocity $=3000 \mathrm{ft} / \mathrm{s}$
}


Ground track and altitude profiles for the nominal missions are shown in Figures $4 \mathrm{a}$ and $4 \mathrm{~b}$. Bank angle and heat rate profiles for the nominal missions are shown individually in Figures 5a51. The peak heat rate objective is $75 \mathrm{BTU} / \mathrm{ft}^{2} / \mathrm{sec}$ for missions $1-3$ and $60 \mathrm{BTU} / \mathrm{ft}^{2} / \mathrm{sec}$ for missions 4-6. Results of 100 dispersed orbital re-entry simulations for each of the missions are shown in Tables 3a through 3f. Dispersion quantities include variables relating to aerodynamic uncertainty, navigation accuracy, and vehicle mass properties, as well as atmospheric density and wind dispersions.

\section{Summary and Conclusions}

This study introduced an automated method for computing orbital re-entry trajectories with heating constraints. The algorithm based on this method, referred to as EGuide, was used successfully as guidance for a variety of sub-orbital and orbital entry missions. By incorporating an initial planning stage to generate trajectories to be flown by a profile-following guidance method, and by reducing the scope of the entry problem to one that is fully characterized with four parameters, the issue of computational complexity is diminished. The unique setup of the orbital re-entry problem provides additional benefits such as smooth control commands to the vehicle and a smooth altitude profile. The use of heat-rate tracking in the initial part of orbital re-entry is particularly effective for two reasons: First, it deals with the orbital re-entry heating constraint directly through the use of feedback control. Second, the computationally efficient heat-rate tracking guidance allows for time to update (in predictor/corrector fashion) the parameters which shape the remainder of the trajectory. 


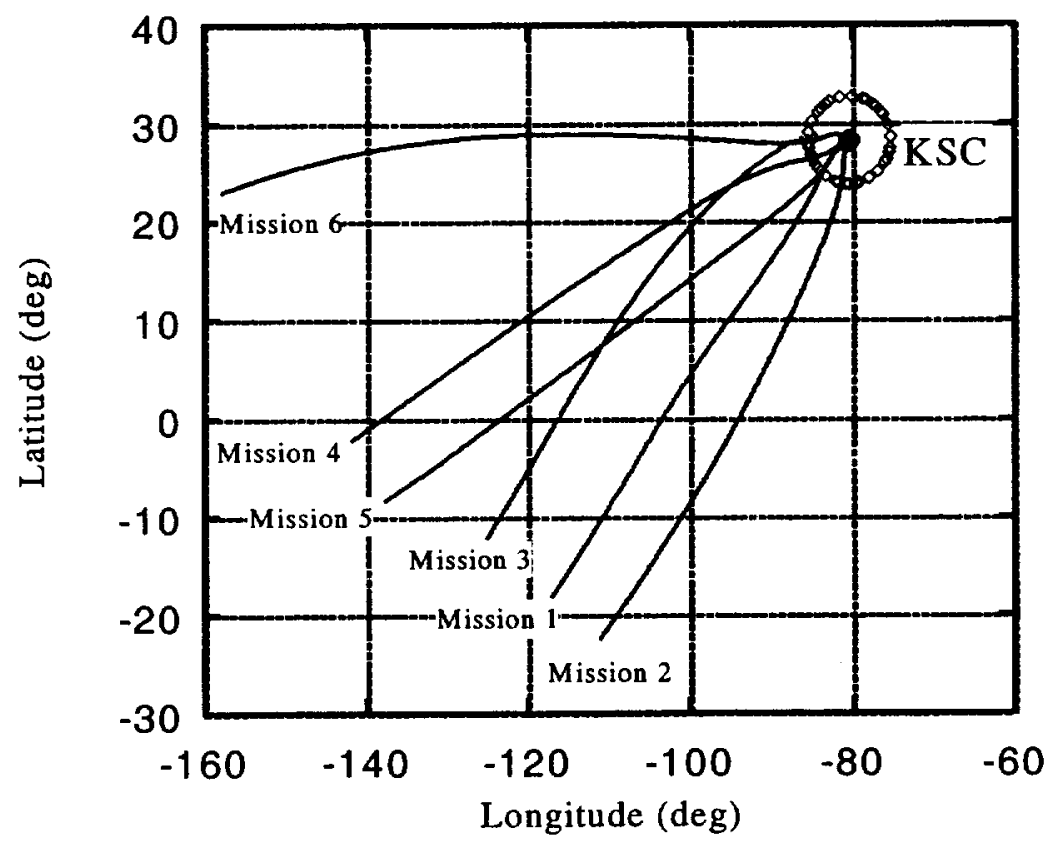

Figure 4a. Missions 1-6 ground tracks

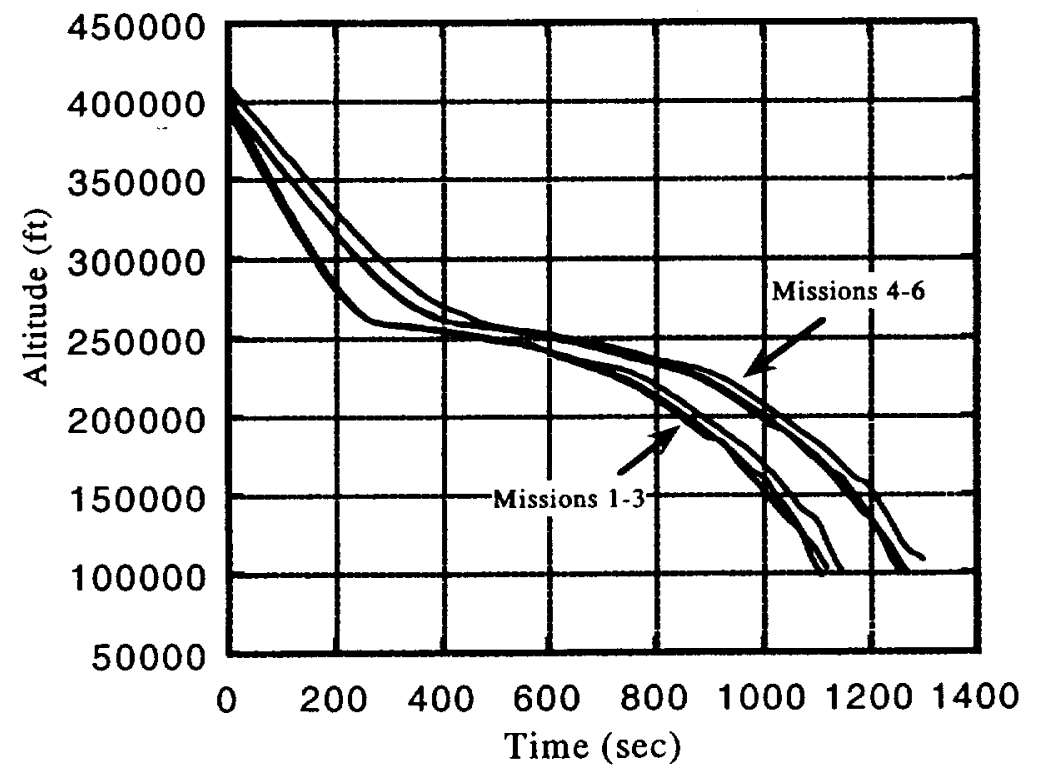

Figure 4b. Missions 1-6 altitude profiles 


\begin{tabular}{|c|c|c|c|c|}
\hline Mission 1 & Min & Max & Average & Stdev \\
\hline Range (nm) & 28.6 & 30.8 & 29.9 & 0.4 \\
\hline Heading (deg) & -11.0 & -7.6 & -9.1 & 0.8 \\
\hline Altitude (ft) & 93163 & 99550 & 95480 & 1006 \\
\hline Max Heat Rate & 64.8 & 74.0 & 67.7 & 1.6 \\
$\left(B T U / \mathrm{ft}^{2} / \mathrm{sec}\right)$ & & 38 & 24.1 & 2.5 \\
\hline Planner Iterations & 22 & 4 & 2.4 & 0.7 \\
\hline Update Iterations & 1 & & & \\
\hline
\end{tabular}

Table 3a. Dispersion results for orbital re-entry mission 1 (100 runs)

\begin{tabular}{|c|c|c|c|c|}
\hline Mission 2 & Min & Max & Average & Stdev \\
\hline Range (nm) & 25.8 & 30.4 & 28.2 & 1.2 \\
\hline Heading (deg) & -10.2 & 10.5 & -3.3 & 6.6 \\
\hline $\begin{array}{c}\text { Altitude (ft) } \\
\text { Max Heat Rate } \\
\text { (BTU/ft'sec) }\end{array}$ & 94816 & 102488 & 97959 & 1969 \\
\hline Planner Iterations & 23 & 75.2 & 69.4 & 1.7 \\
\hline Update Iterations & 1 & 27 & 25.5 & 0.9 \\
\hline
\end{tabular}

Table 3b. Dispersion results for orbital re-entry mission 2 (100 runs) 


\begin{tabular}{|c|c|c|c|c|}
\hline Mission 3 & Min & Max & Average & Stdev \\
\hline Range (nm) & 24.5 & 30.9 & 28.5 & 1.6 \\
\hline Heading (deg) & -24.1 & 10.9 & -5.5 & 5.5 \\
\hline Altitude (ft) & 94769 & 105245 & 99396 & 2472 \\
\hline $\begin{array}{c}\text { Max Heat Rate } \\
\left(B T U / \mathrm{ft}^{2} / \mathrm{sec}\right)\end{array}$ & 64.2 & 72.6 & 66.9 & 1.4 \\
\hline Planner Iterations & 63 & 104 & 90.5 & 11.6 \\
\hline Update Iterations & 1 & 6 & 2.6 & 0.9 \\
\hline
\end{tabular}

Table 3c. Dispersion results for orbital re-entry mission 3 (100 runs)

\begin{tabular}{|c|c|c|c|c|}
\hline Mission 4 & Min & Max & Average & Stdev \\
\hline Range (nm) & 28.2 & 32.0 & 30.7 & 0.7 \\
\hline $\begin{array}{c}\text { Heading (deg) } \\
.\end{array}$ & -8.5 & 10.5 & 7.7 & 4.2 \\
\hline Altitude (ft) & 97579 & 102521 & 99372 & 1128 \\
\hline Max Heat Rate & 59.6 & 68.6 & 62.2 & 1.6 \\
\hline BTU/ft $\left.{ }^{2} / \mathrm{sec}\right)$ & 18 & 23 & 19.9 & 1.4 \\
\hline Planner Iterations & 1 & 4 & 2.5 & 0.6 \\
\hline Update Iterations & & & & \\
\hline
\end{tabular}

Table 3d. Dispersion results for orbital re-entry mission 4 (100 runs) 


\begin{tabular}{|c|c|c|c|c|}
\hline Mission 5 & Min & Max & Average & Stdev \\
\hline Range (nm) & 26.2 & 31.0 & 28.6 & 1.3 \\
\hline Heading (deg) & -10.3 & 9.2 & -4.0 & 5.7 \\
\hline Altitude (ft) & 97071 & 106301 & 100889 & 2177 \\
\hline $\begin{array}{c}\text { Max Heat Rate } \\
\left.\text { (BTU/ft }{ }^{2} / \mathrm{sec}\right)\end{array}$ & 57.8 & 64.8 & 60.5 & 1.3 \\
\hline Planner Iterations & 22 & 27 & 23.2 & 1.2 \\
\hline Update Iterations & 1 & 4 & 2.4 & 0.7 \\
\hline
\end{tabular}

Table 3e. Dispersion results for orbital re-entry mission 5 (100 runs)

\begin{tabular}{|c|c|c|c|c|}
\hline Mission 6 & Min & Max & Average & Stdev \\
\hline Range (nm) & 26.3 & 31.1 & 29.3 & 1.2 \\
\hline Heading (deg) & -10.4 & 6.8 & -7.0 & 3.4 \\
\hline $\begin{array}{c}\text { Altitude (ft) } \\
\text { Max Heat Rate } \\
\left(B T U / \mathrm{ft}^{2} / \mathrm{sec}\right)\end{array}$ & 96493 & 104782 & 99252 & 1894 \\
\hline Planner Iterations & 58.3 & 67.6 & 61.5 & 1.7 \\
\hline Update Iterations & 1 & 3 & 22.6 & 0.6 \\
\hline
\end{tabular}

Table 3f. Dispersion results for orbital re-entry mission 6 (100 runs) 


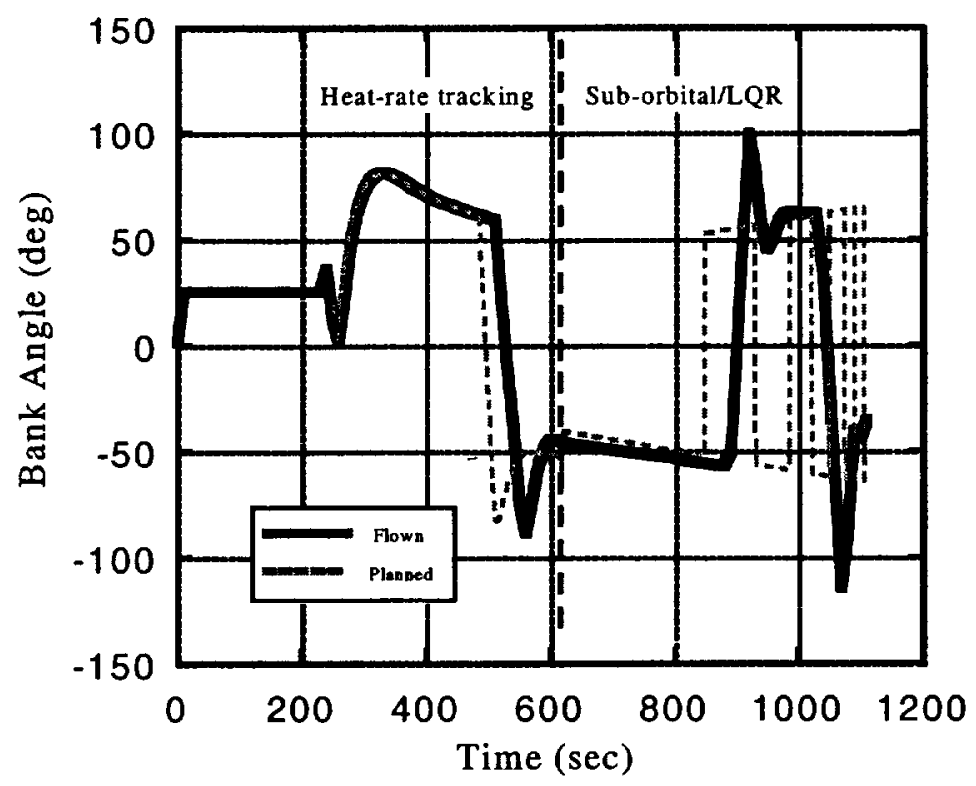

Figure 5a Mission 1 bank angle profile

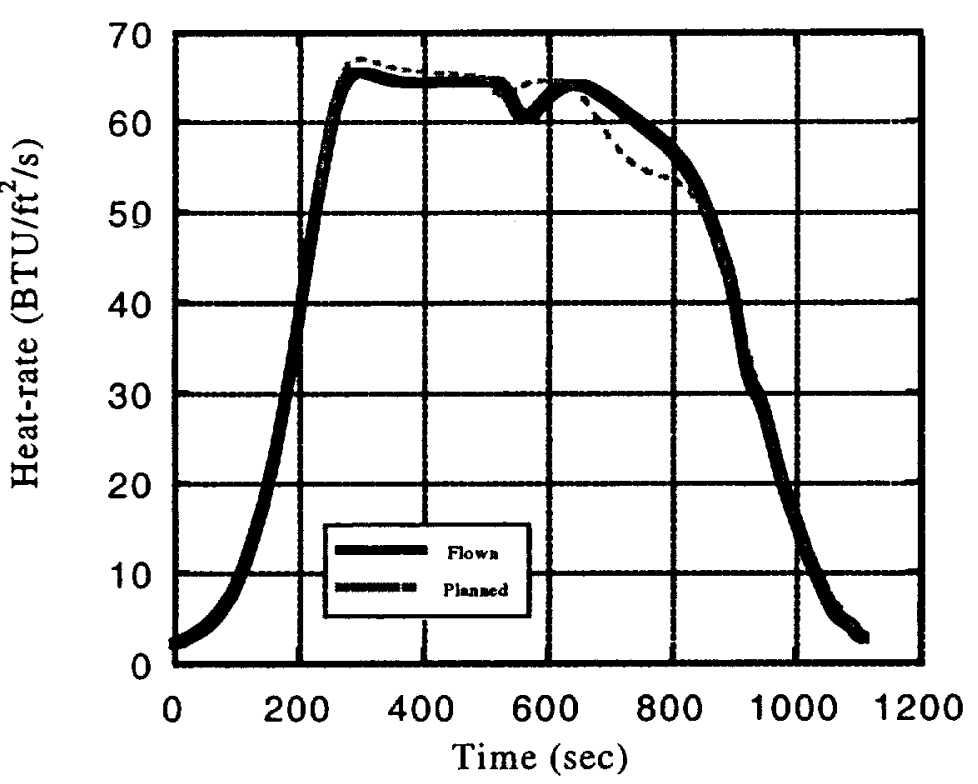

Figure 5b Mission 1 heat-rate profile 


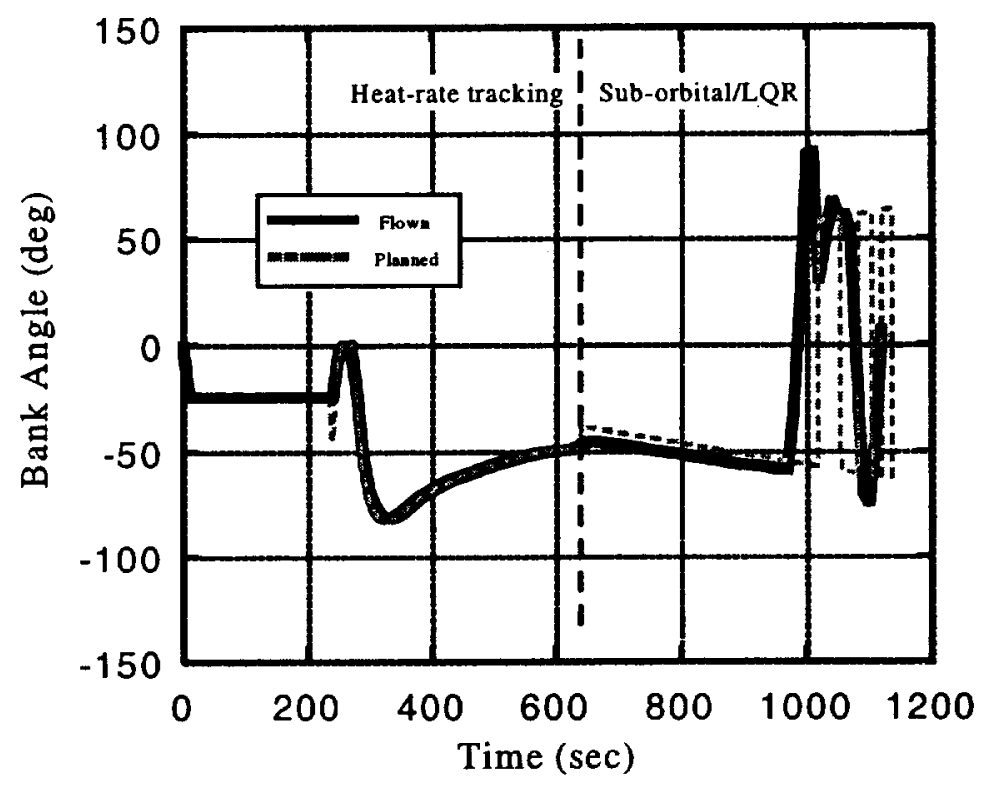

Figure 5c Mission 2 bank angle profile

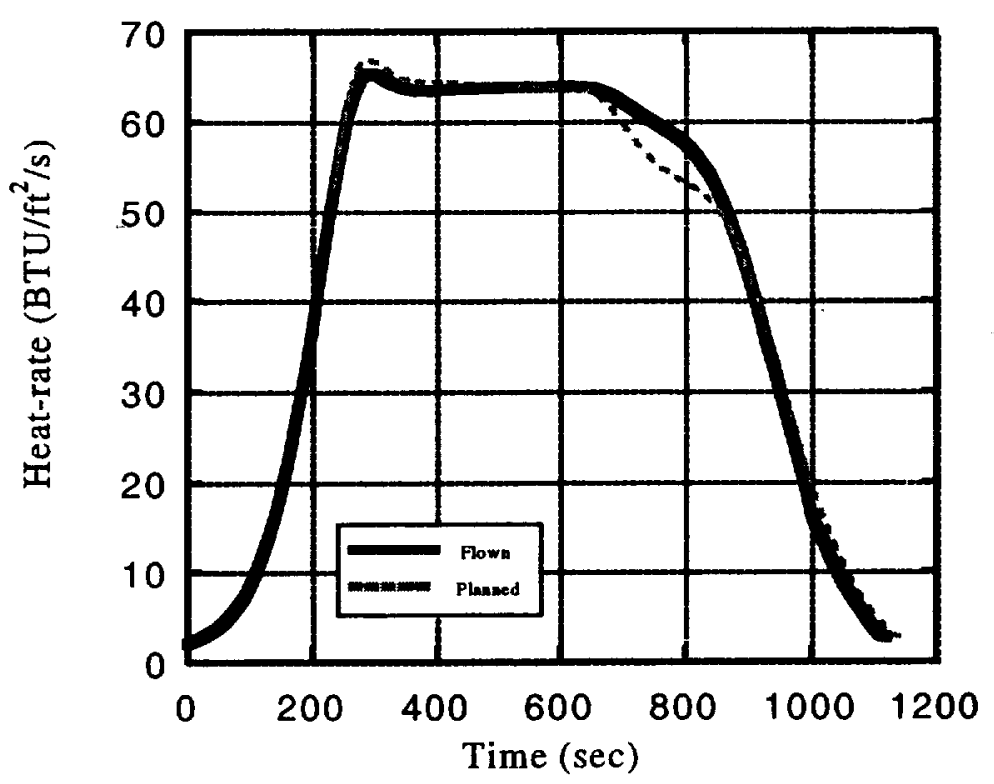

Figure 5d Mission 2 heat-rate profile 


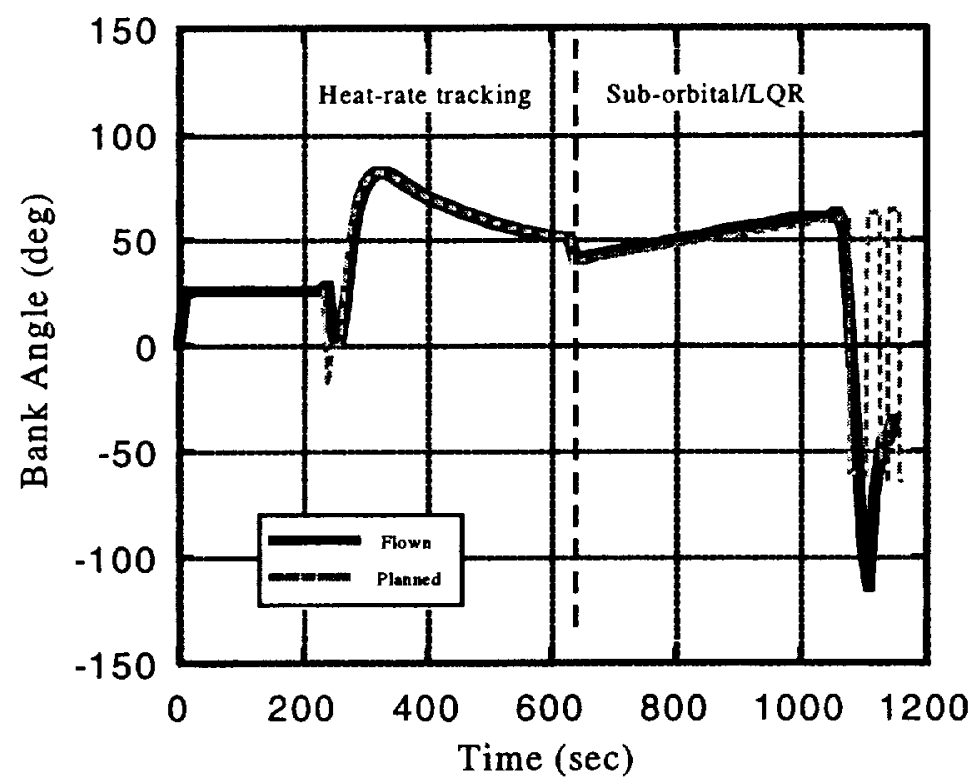

Figure 5e Mission 3 bank angle profile

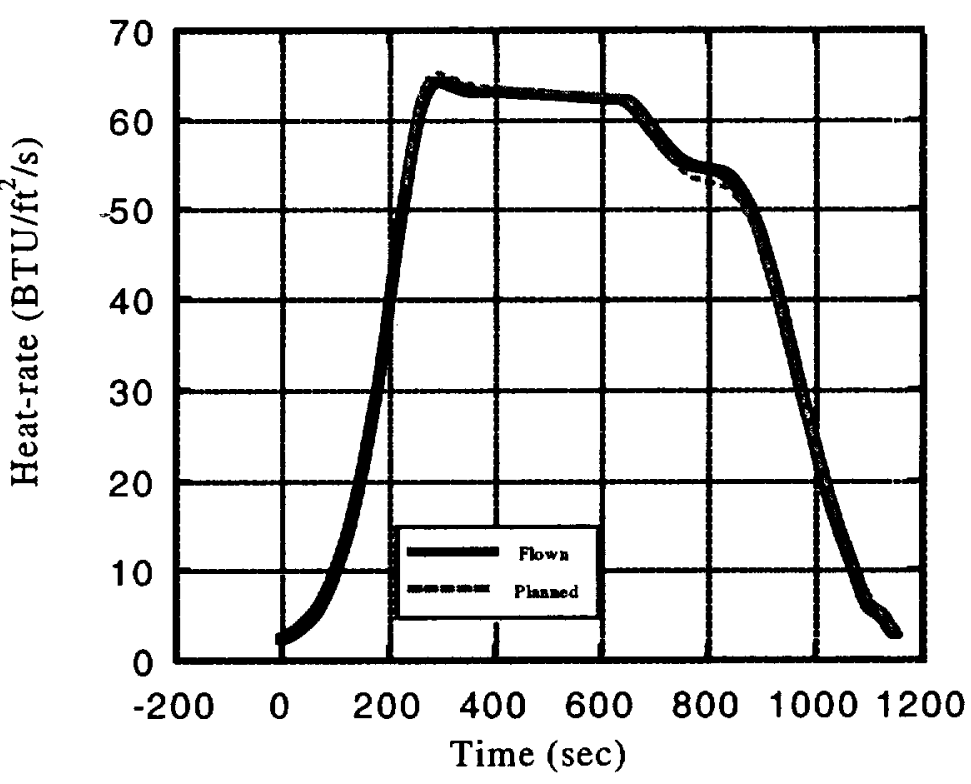

Figure $5 f$ Mission 3 heat-rate profile 


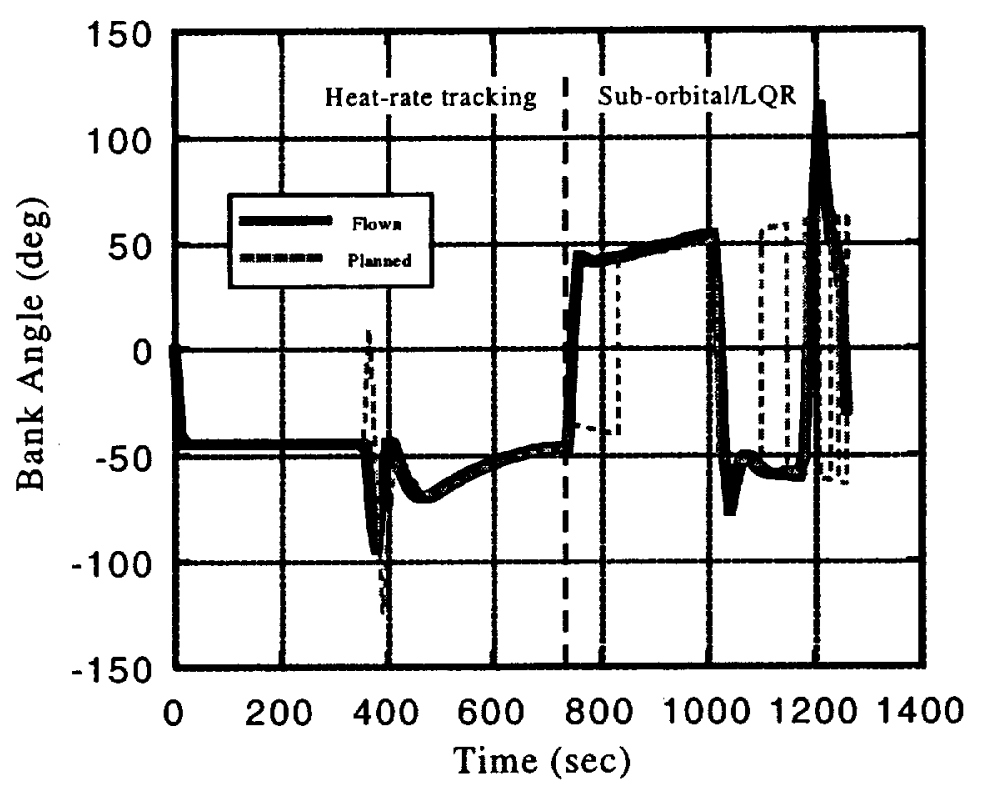

Figure 5g Mission 4 bank angle profile

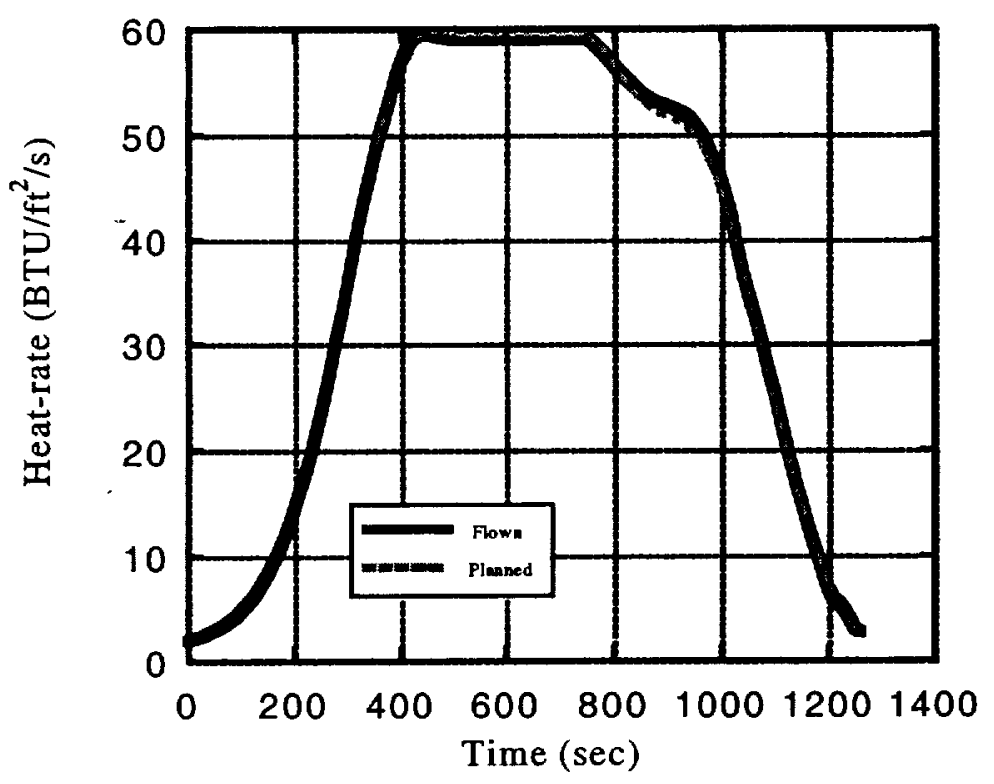

Figure 5h Mission 4 heat-rate profile 


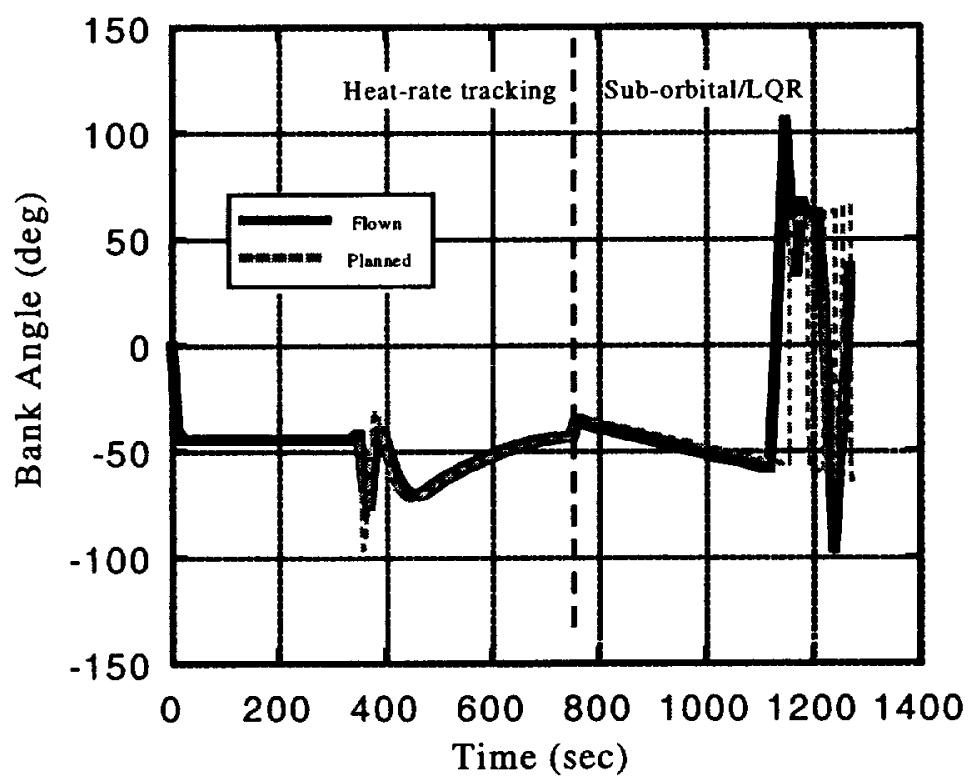

Figure 5i Mission 5 heat-rate profile

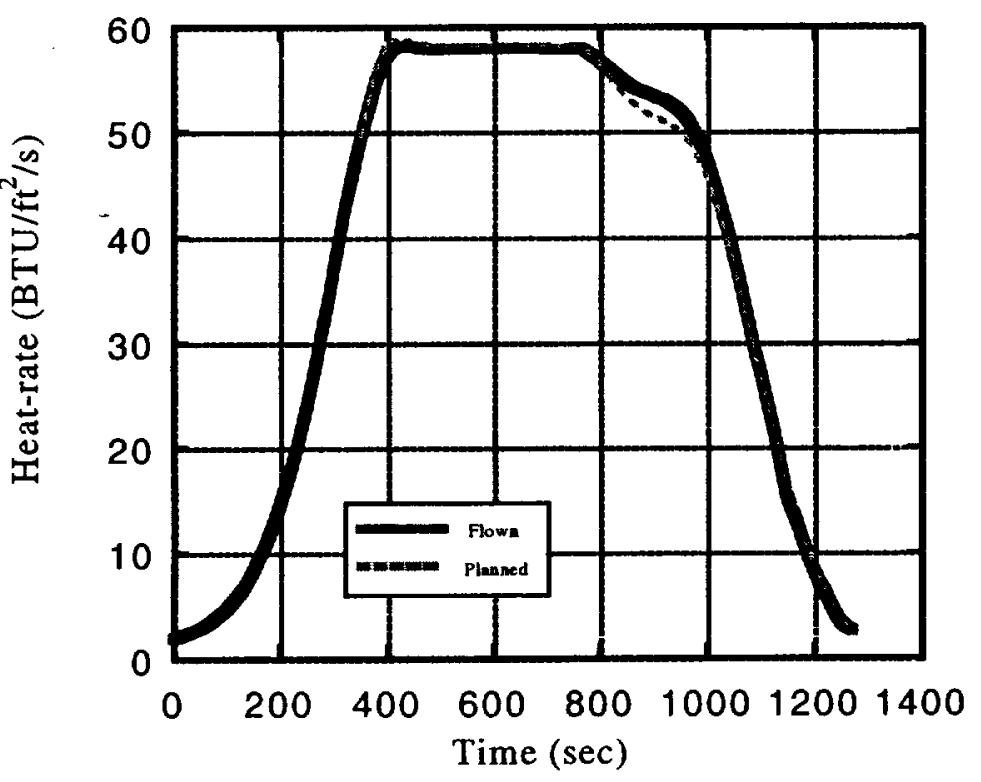

Figure 5j Mission 5 heat-rate profile 


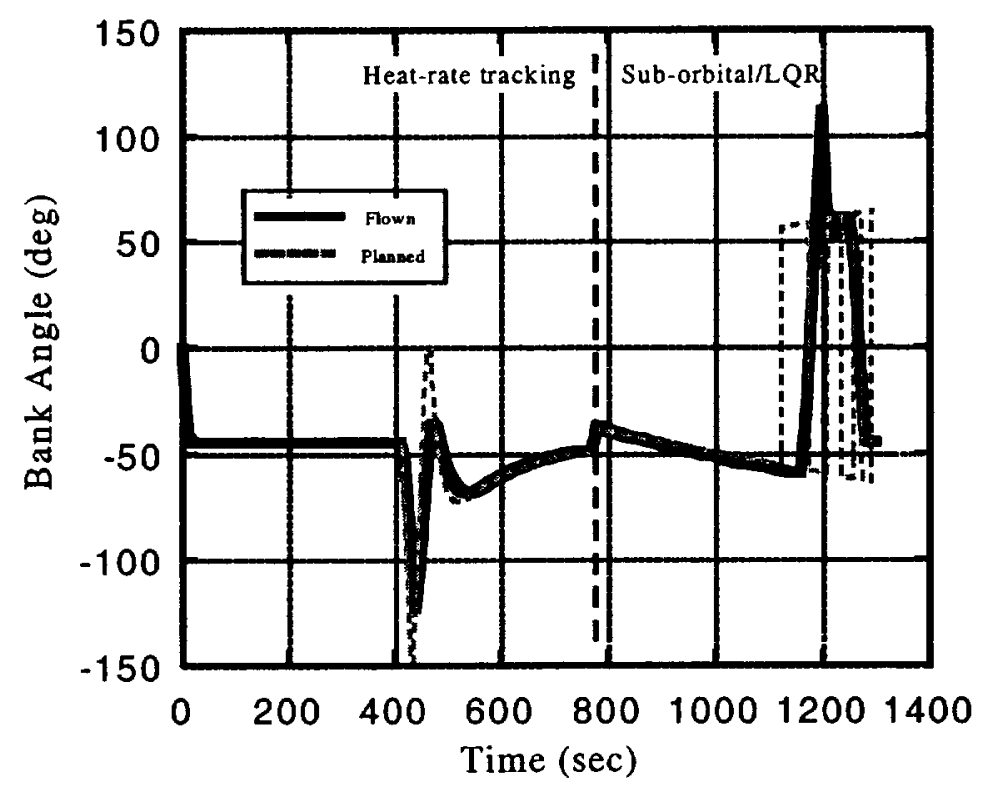

Figure 5k Mission 6 heat-rate profile

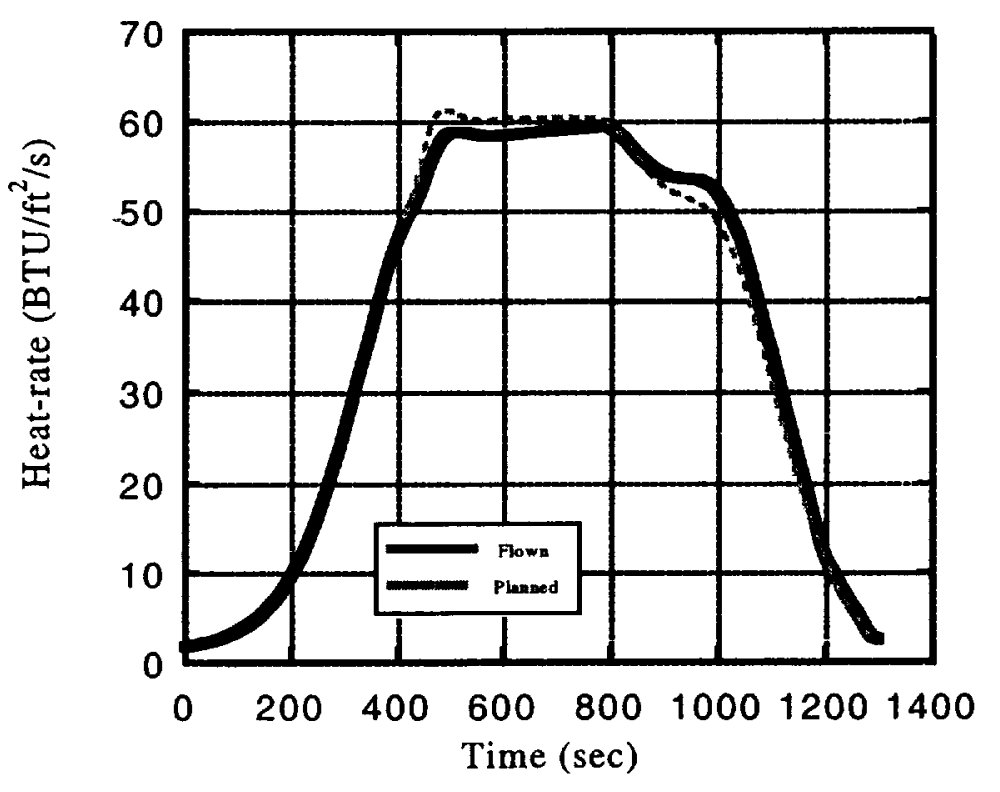

Figure 5l Mission 6 heat-rate profile 


\section{References}

[1] Harpold, J.C., and Graves, C.A. Jr., "Shuttle Entry Guidance," Journal of the Astronautical Sciences, Vol. XXVII, No. 3, pp 239-267, July-September, 1979.

[2] Powell, R.W. "Numerical Roll Reversal Predictor-Corrector Aerocapture and Precision Landing Guidance Algorithms for the Mars Surveyor Program 2001 Missions," AIAA 19984574 August 1998.

[3] Spratlin, K.M. "An Adaptive Numeric Predictor-Corrector Guidance Algorithm for Atmospheric Entry Vehicles," M.S. Thesis - MTT, Cambridge.

[4] Youssef, H., Chowdhry, R., Lee, H., Rodi, P., and Zimmerman, C., "Predictor-Corrector Entry Guidance for Re-usable Launch Vehicles" AIAA 2001-4043. August 2001

[5] Dukeman, G.A., "Profile following Entry Guidance using Linear Quadratic Regulator Theory", AIAA 2002-4457.

[6] Press, W.H., Teukolsky, S.A., Vetterling, W.H., and Flannery, B.P., "Numerical Recipes in C." Second Edition., Cambridge Univ. Press, New York, 1993, pp 379-389. 
[7] Hanson, J., Jones, R., and Krupp, D., “Advanced Guidance and Control Methods for Reusable Launch Vehicles: Test Results," AIAA 2002-4561. AIAA Guidance, Navigation, and Control Conference, Monterey, CA, Aug 2002.

[8] Vinh, N.X., “Optimal Trajectories in Atmospheric Flight”, Elsevier, New York, 1981, pp 4762.

[9] Vinh, N.X., et al., Hypersonic and Planetary Entry Flight Mechanics, The University of Michigan Press, Ann Arbor, MI, 1980. 